\title{
SPARCE: Sparsity aware General Purpose Core Extensions to Accelerate Deep Neural Networks
}

\author{
Sanchari Sen, Shubham Jain, Swagath Venkataramani, Anand Raghunathan
}

\begin{abstract}
Deep Neural Networks (DNNs) have emerged as the method of choice for solving a wide range of machine learning tasks. Satiating the enormous growth in computational demand posed by DNNs is a key challenge for computing system designers and has most commonly been addressed through the design of custom accelerators. However, these specialized accelerators that utilize large quantities of multiply-accumulate units and on-chip memory are prohibitive in many design scenarios (e.g., wearable devices and loT sensors), due to the stringent area/cost constraints. Therefore, accelerating DNNs on these low-power systems, comprising of mainly the indispensable general-purpose processor (GPP) cores, requires new approaches. In this work, we focus on improving the performance of DNNs on GPPs by exploiting a key attribute of DNNs, i.e. sparsity. We propose Sparsity aware Core Extensions (SPARCE) - a set of micro-architectural and ISA extensions that leverage sparsity and are minimally intrusive and low-overhead. We address the key challenges associated with exploiting sparsity in GPPs, viz., dynamically detecting whether an operand (e.g., the result of a load instruction) is zero and subsequently skipping a set of future instructions that use it. To maximize performance benefits, our design ensures that the instructions to be skipped are prevented from even being fetched, as squashing instructions comes with a penalty (e.g., a pipeline stall). SPARCE consists of 2 key micro-architectural enhancements. First, a Sparsity Register File (SpRF) is utilized to track registers that are zero. Next, a Sparsity aware Skip Address (SASA) table is used to indicate instruction sequences that can be skipped, and to associate specific SpRF registers to trigger instruction skipping. When an instruction is fetched,

SPARCE dynamically pre-identifies whether the following instruction(s) can be skipped, and if so appropriately modifies the program counter, thereby skipping the redundant instructions and improving performance. We model SPARCE using the gem5 architectural simulator, and evaluate our approach on 6 state-of-the-art image-recognition DNNs in the context of both training and inference using the Caffe deep learning framework. On a scalar microprocessor, SPARCE achieves $1.11 \times-1.96 \times$ speedups across both convolution and fully-connected layers with 10\%-90\% sparsity, and 19\%-31\% reduction in execution time at the overall application-level. We also evaluate SPARCE on a 4-way SIMD ARMv8 processor using the OpenBLAS library, and demonstrate that SPARCE achieves $8 \%-15 \%$ reduction in the application-level execution time.
\end{abstract}

Index Terms—Deep Learning, Deep Neural Networks, Sparsity, General Purpose Processors

\section{INTRODUCTION}

Deep neural networks (DNNs) have revitalized the field of machine learning by achieving accuracy levels beyond human perception in a variety of image, video, text and speech processing tasks [1]-[8]. Today, they have emerged as the de facto solution approach for challenging artificial intelligence problems and are deployed in several products and services, including Google's image and voice search [9], Facebook's DeepFace [10] and DeepText [11], Apple's Siri [12], to name a few. However, one of the key challenges to the ubiquitous adoption of DNNs is their computational demand, which far outstrips the capabilities of modern computing platforms. For example, ResNet-152 [1], a state-of-the-art DNN for image recognition, requires $\sim 11.3$ giga operations to classify a single $224 \times 224$ image. As DNNs begin to pervade the spectrum of computing devices from high performance servers to low-power edge/IoT devices, the demands on compute efficiency are expected grow even more stringent. Consequently, there is an urgent need to explore new approaches to improve their implementation efficiency.

- Sanchari Sen, Shubham Jain and Anand Raghunathan are with School of Electrical and Computer Engineering, Purdue University, West Lafayette, IN, 47906, USA.

E-mail: $\{$ sen9,jain130,raghunathan $\} @$ purdue.edu

- Swagath Venkataramani is with IBM T.J Watson Research Center, Yorktown Heights, $N Y$.

E-mail: swagath.venkataramani@ibm.com
Realizing this need, prior research efforts have explored several key directions. A majority of research efforts exploit the compute and communication characteristics of DNNs to efficiently parallelize them on distributed platforms [13]-[19] or design specialized accelerator architectures [20]-|31]. Other efforts leverage the intrinsic error resilience of DNNs to approximate selected computations [32]-[39] or utilize non-CMOS technologies whose device characteristics match the compute kernels of DNNs [40][42].

Complementary to the above efforts, we focus on a different design scenario and target deeply embedded systems such as wearables and IoT edge devices where the additional area/ cost imposed by custom-accelerators is prohibitive. For example, even a low power DNN accelerator such as Eyeriss [31] occupies $12.25 \mathrm{~mm}^{2}$ area which is $30 \times$ larger than the $0.4 \mathrm{~mm}^{2}$ occupied by an ultra low power Cortex A35 core [43]. Accelerating DNNs on these low-power systems then comes down to accelerating them on the indispensable general-purpose processor (GPP) cores already present in these systems. We focus on improving the execution time of DNNs on GPPs by leveraging sparsity in different DNN data-structures, viz., features, weights and backpropagated errors. Sparsity in DNNs can be both static or dynamic depending on whether the zero values remain constant or vary across different inputs to the network. Sparsity in weights, introduced by pruning connections in the network after training, is static in nature. In contrast, feature and error sparsities, caused by the thresholding nature of the ReLU (Rectified Linear Unit) activation functions, are dynamic in nature. 
Prior efforts that exploit sparsity to accelerate DNNs can be grouped into two classes based on whether they exploit static or dynamic sparsity, as shown in Figure 1 . Specialized accelerators [31], [44]-[48] have been proposed to exploit both forms of sparsity. However, these techniques are closely tied to a given accelerator architecture thereby preventing their applicability to GPPs. In contrast, static sparsity in weights can be exploited on GPPs through software-only approaches [49]-[51] that involve sparse encodings and sparse matrix multiplication routines. However, extending them to exploit the intermediate levels of dynamic sparsity $(40 \%-70 \%)$ can be counter-productive because of the encoding overhead involved at runtime. For example, the sparse encoding overhead involved in performing sparseBLAS based matrix multiplication on the sparse conv3 layer features of AlexNet [52] causes a slowdown of $2.78 \times$. We observe across 6 image-recognition DNNs that dynamic sparsity in features results in $45.1 \%$ of the computations being rendered redundant during inference, presenting a significant opportunity for improving performance. We believe our effort, SPARCE, is the first to successfully exploit dynamic sparsity in DNNs on GPPs. To that end, we propose micro-architectural and ISA extensions that are minimally intrusive and low-overhead. Moreover, since static sparsity is a special case of dynamic sparsity where the location of zeros remains constant across inputs, the extensions also allow SPARCE to exploit static sparsity in weights.

\begin{tabular}{|c|c|c|}
\hline & Accelerators & General Purpose Processors \\
\hline 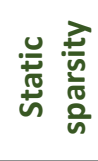 & $\begin{array}{c}\text { Eyeriss[31], } \\
\text { EIE[45], SCNN[46], } \\
\text { Cambricon-x[47], } \\
\text { GPU ZeroSkip[48] }\end{array}$ & $\begin{array}{l}\text { SparseCNN[49], SSL[50], } \\
\text { Scalpel[51], } \underline{\text { SPARCE }}\end{array}$ \\
\hline 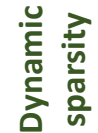 & $\begin{array}{c}\text { Eyeriss[31], } \\
\text { Cnvlutin[44], } \\
\text { EIE[45], SCNN[46] }\end{array}$ & $\underline{\text { SPARCE }}$ \\
\hline
\end{tabular}

Fig. 1. Related work: Exploiting sparsity in DNNs

To exploit dynamic sparsity, GPPs need to be equipped to dynamically detect if the result of an instruction (e.g., a load from a sparse data structure) is zero and if so, skip a set of future instructions that use its result. However, there are two key challenges: (i) the instructions to be skipped often do not immediately follow the instruction that determines whether they may be; moreover, the instructions to be skipped may be noncontiguous in the program, and (ii) to maximize performance benefits, the instructions to be skipped should be prevented from even being fetched, as squashing the instruction in-flight would diminish the benefits by introducing a pipeline stall.

Sparsity aware Core Extensions (SPARCE). To overcome the aforementioned challenges, we propose Sparsity aware Core Extensions (SPARCE), comprised of two key micro-architectural enhancements. First, SPARCE contains a Sparsity Register File (SpRF) to track which general purpose registers that contain a zero. We achieve this by augmenting the writeback stage of the processor to check if the update to a register is zero and appropriately modify the corresponding SpRF entry. Next, a Sparsity Aware Skip Address (SASA) table is used to store instruction sequences and the conditions under which they can be skipped i.e., the registers in the SpRF that need to be zero for the instructions to become redundant. Whenever SPARCE fetches an instruction, it uses the SASA table and the SpRF to pre-identify whether the following instruction(s) can be skipped. If so, the program counter is modified to directly fetch the next irredundant instruction.

In summary, the key contributions of this work are:

- We propose Sparsity aware Core Extensions (SPARCE) to accelerate DNNs on general purpose processors by skipping redundant computations borne out of sparsity in the different DNN data-structures.

- SPARCE comprises of micro-architectural enhancements to dynamically track when the result of an instruction is zero, pre-identify future instructions that are rendered redundant, and prevent them from being fetched and executed, thereby improving performance.

- We evaluate SPARCE on a suite of 6 state-of-the-art imagerecognition DNN benchmarks using the Caffe deep learning framework. We achieve application-level speedups of $19 \%$ $31 \%$ on low-power embedded scalar microprocessors. We also achieve speedups of $8 \%-15 \%$ over highly optimized baseline implementations that use OpenBLAS on an ARMv8 processor with 4-way SIMD and prefetching support.

The rest of the paper is organized as follows. Section 2 explains the sources of sparsity in DNNs. Section 3 details the key design principles of SPARCE and demonstrates them in the context of an in-order pipelined processor. Section 4 shows SPARCE in action using the ARM-BLAS GEMM routine as a case study. Section 5 describes the experimental methodology and the results are presented in Section 6. Section 7 presents related research efforts and Section 8 concludes the paper.

\section{SPARSITY IN DNNS: SOURCES AND OPPORTU- NITY}

In this section, we first provide a brief background on DNNs. We then explain the various static and dynamic sources of sparsity in the different DNN data-structures, and quantify the opportunity for performance improvement afforded by sparsity.

\subsection{DNN: Background}

DNNs are networks of primitive compute units called neurons, organized into layers. Each layer is associated with a set of parameters called weights. DNNs operate in two phases viz. training and inference. During the training phase, a labelled training dataset is used to iteratively refine the weights of the DNN. In the inference phase, the trained DNN is used to classify new inputs.

Computationally, DNN executions iteratively perform 3 key steps viz. Forward Propagation (FP), Backpropagation (BP), and Weight Gradient and Update (WG), that operate on 4 datastructures, viz., features, weights, errors and gradients. All three steps (FP, BP, WG) are performed during the training phase, while inference involves only the FP step. In FP, inputs to the DNN are propagated through its layers to produce the DNN outputs. In each layer, the input features are operated on with its weights to produce its output features, which are then fed to the next layer and so on. In $\mathrm{BP}$, errors observed at the output of the DNN are propagated backwards through each layer of the DNN. In this case, the error at the output of a layer is operated on with its weights to compute the error at its inputs. In WG, the input features and the output error of each layer are used to refine its weights. 


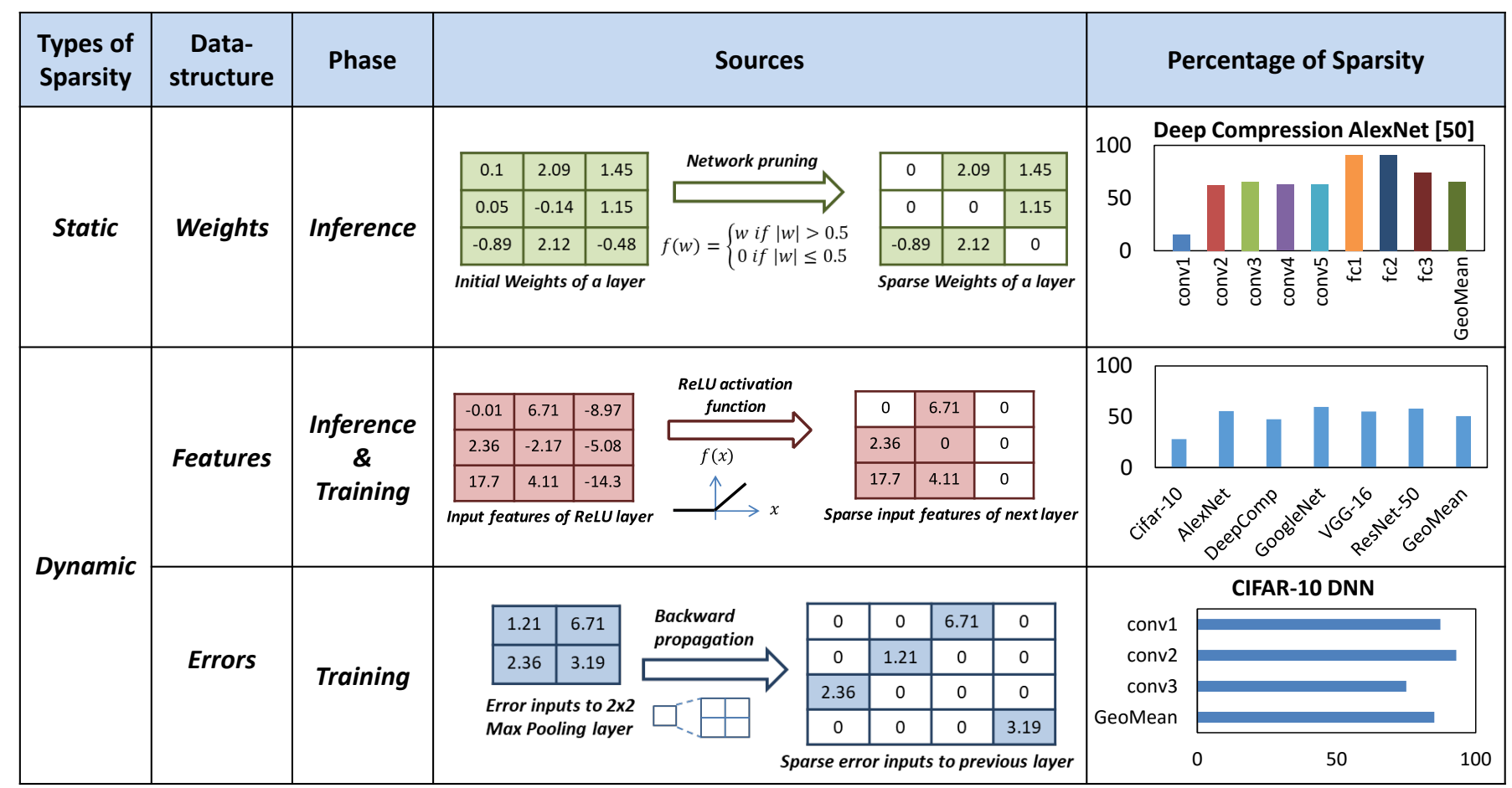

Fig. 2. Different forms of sparsity in DNNs

\subsection{Sources of Sparsity in DNNs}

In practice, all major DNN data-structures - features, weights, errors and gradients - exhibit significant levels of sparsity, which can be exploited for computational savings. Three of the four data-structures (all except weight gradients) are used as inputs to multiply-and-accumulate operations in the different steps (FP/BP/WG), which become redundant when one of the input operands is zero. Among these three sparse multiply-andaccumulate operands, weights exhibit static sparsity that remains constant across different inputs while the remaining two datastructures (features and errors) exhibit dynamic sparsity that varies dynamically across different inputs. Figure 2 summarizes the sparsity in the different DNN data-structures, which we describe in the remainder of this subsection.

\subsubsection{Static Sparsity}

Weight Sparsity. Sparsity in weights occurs during the inference phase of the DNN. As shown in Figure 2 after training, connections whose weights are close to zero are pruned to compress the model size [26], [36], [37]. The last column in Figure 2] shows the fraction of zero weights in the different layers of the AlexNet model trained using deep compression [36]. We find the sparsity to vary between $18 \%-85 \%$ across the different layers. Weight sparsity is static in nature because of the fact that zero weights are identified before the inference phase.

\subsubsection{Dynamic Sparsity}

Feature sparsity. Sparsity in features stems from the thresholding nature of the activation function present at the output of each layer. As shown in Figure 2, the predominantly used ReLU (Rectified Linear Unit) activation function clips negative inputs to zero. Figure 2 also shows the average feature sparsity exhibited by various DNN benchmarks, which ranges between $\sim 25 \%-60 \%$. Feature sparsity has a dynamic nature i.e., because neurons whose outputs are zero vary considerably across inputs. Figure 3 illustrates this property using feature maps obtained at the conv3 of AlexNet, produced by two different inputs from the ImageNet dataset. We find the spatial variation in white pixels, which indicate zero output, to be substantial across inputs.

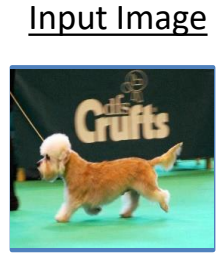

Input 1

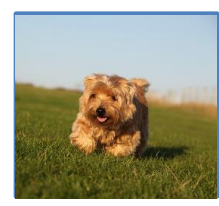

Input 2
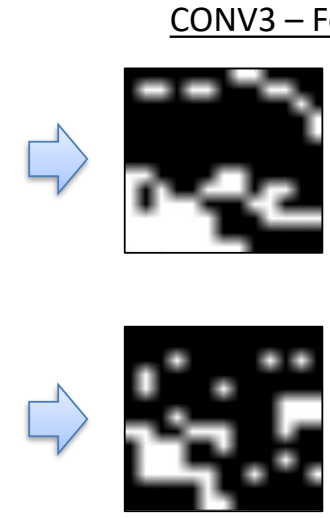

Feature: 1

\section{CONV3 - Feature Map}
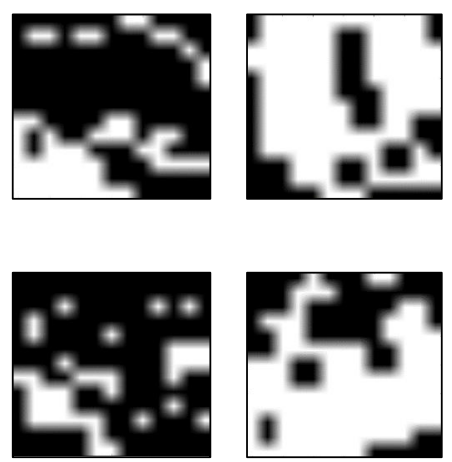

Feature: 100
Fig. 3. Variation in feature sparsity of AlexNet CONV3 layer across input images

Error Sparsity. Sparsity in the error data-structure originates from two sources. First, the derivative of the activation function, such as ReLU, is zero when the error at the output of the layer is negative. Next, when errors are propagated back through a maxpooling layer, as shown in Equation 1 . only one input of each pooling window is set a non-zero error value.

$\frac{\partial E}{\partial y_{l}}(x+p, y+q)= \begin{cases}0, & \text { if } y_{l+1}(x, y) \neq y_{l}(x+p, y+q) \\ \frac{\partial E}{\partial y_{l+1}}, & \text { otherwise }\end{cases}$ 
For example, if a pooling window of size $2 \times 2$ is used, at least three quarters of the error values are sparse. Similar to feature sparsity, the error sparsity is also dynamic. The average error sparsity in different layers of a CIFAR-10 DNN is shown in Figure 2

\subsection{Opportunity for Computational Savings}

Figure 4 shows the fraction of multiplyaccumulate (MAC) computations that are rendered redundant for each DNN benchmark due to dynamic sparsity in features during inference. We find that between 25\%-60\% (average: $45 \%$ ) of the computations can be skipped, underscoring the substantial opportunity

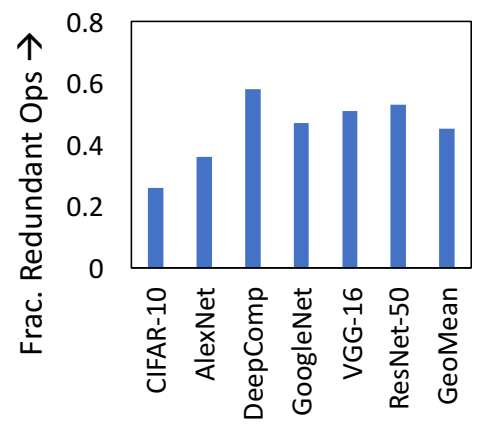

Fig. 4. Redundant ops for performance improvement. Figure 5 shows how the fraction of redundant operations varies across 1000 different inputs for the AlexNet DNN. We observe $\sim 14 \%$ variation across inputs, although each input shows considerable opportunity for reduction in execution time (minimum: $28 \%$ ).

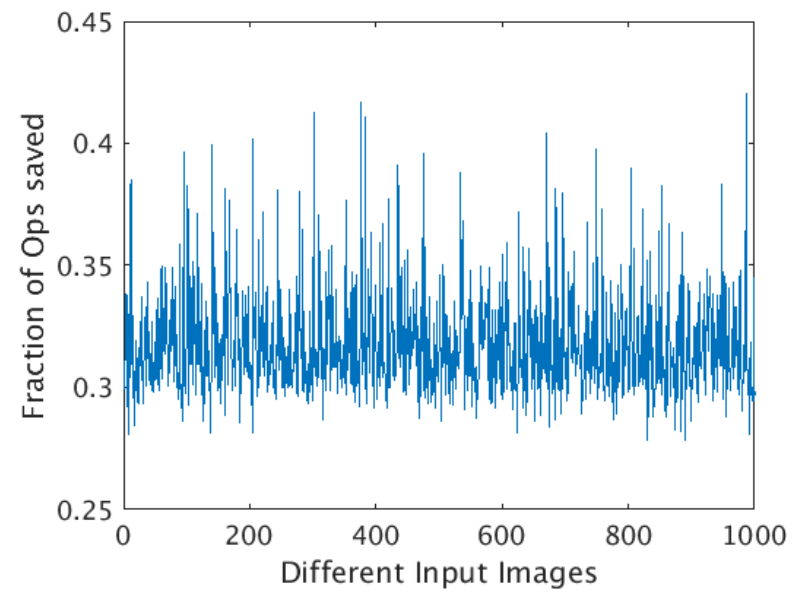

Fig. 5. Fraction of redundant ops across different inputs of AlexNet

In summary, the dynamic sparsity present in the feature and error data-structures offers a substantial opportunity to accelerate DNNs. However, the levels of sparsity are not extreme enough to completely exploit them in software, and this coupled with their dynamic nature necessitates hardware solutions to realize benefits in the context of general purpose processors.

\section{Sparce: Sparsity Aware General Purpose Core Extensions}

To exploit the different forms of sparsity and improve DNN performance on GPPs, we propose Sparsity aware Core Extensions (SPARCE), a set of micro-architectural and ISA extensions that are general-purpose, minimally intrusive and low-overhead. In this section, we present the key ideas behind SPARCE and describe how they can be integrated within an in-order processor pipeline.

\subsection{Challenges}

The key challenge in exploiting sparsity is to equip the processor with the ability to dynamically detect if the result of an instruction is zero and if so, skip a list of future instructions that are rendered redundant. We illustrate this challenge using the assembly code snippet shown in Figure 6, which computes the dot-product of two vectors, $I N P$ and $K E R$, each of size $N$, to produce a scalar OUT. Registers $r 0, r 1$ and $r 2$ hold the data operands, while $p 0, p 1$ and $p 2$ are pointers that hold their respective memory locations. For each instruction in the program, Figure 6 shows the instructions that can be skipped when its result is zero. For example, when the INP load returns a zero (Inst. 2), the subsequent $K E R$ load (Inst. 4), and the multiply and add instructions can be skipped (Insts. 6-7). It is noteworthy that the computational savings is a weighted sum of the number of instructions skipped and the cycles taken by each instruction. For instance, floating point multiply and add instructions may take 3-5 cycles to execute, while a load incurs variable cycles depending on the level of cache hierarchy accessed.

\begin{tabular}{|c|c|c|c|}
\hline \multicolumn{2}{|r|}{ Vector Dot-product } & \multicolumn{2}{|c|}{$\begin{array}{l}\text { Redundant ins } \\
\text { if result }==0\end{array}$} \\
\hline \multirow{10}{*}{ LOOP } & LD r2, [p2] //Load OUT & (1) & -- \\
\hline & LD r0, [p0] //Load INP & (2) & $4,6-7$ \\
\hline & ADD p0, p0, \#I & (3) & --- \\
\hline & LD rl, [pI] //Load KER & (4) & $6-7$ \\
\hline & ADD pl, pl, \#l & (5) & --- \\
\hline & FMUL $\mathrm{r} 3, \mathrm{rl}, \mathrm{r} 0 / / \mathrm{r} 3=$ INP*KER & (6) & 7 \\
\hline & FADD r2, r2, r3 // OUT += r3 & (7) & --- \\
\hline & INC INDEX & (8) & --- \\
\hline & BNE INDEX, \#N, LOOP & (9) & --- \\
\hline & ST r2, [p2] & $(10)$ & --- \\
\hline
\end{tabular}

Fig. 6. Redundant instructions due to sparsity in vector dot-product evaluation

The following observations highlight the challenges in detecting and benefiting from sparsity.

- Location of redundant instructions. In a program, the instructions that can be skipped may not immediately follow the instruction producing the zero result. Worse, redundant instruction sequences may be scattered non-contiguously through the program. For instance, in the program shown in Figure 6, when inst. 2 returns a zero, 2 non-contiguous instruction sequences (Inst. 4 and Insts. 6-7) need to skipped. Hence, efficient ways to capture which instructions can be skipped is key to leveraging sparsity.

- Avoiding redundant instruction fetches. To maximize performance, the instructions that can be skipped need to be prevented from even being fetched. This is especially critical in the context of in-order pipelines, where even if the instruction is squashed after being fetched, it would introduce a bubble in the pipeline. For example, if the condition for $r 0$ or $r 1$ being zero is checked after the FMUL instruction is fetched (Inst. 6), it would result in a bubble flowing through the pipeline in place of Inst. 6. It is worth noting that, in the context of multi-cycle instructions, squashing the instruction after it is fetched can still improve performance. 
- Use of SIMD instructions. GPPs use vector units with SIMD (Single Instruction, Multiple Data) execution engines to exploit fine-grained data parallelism in workloads. SIMD instructions can be skipped only if computations performed on all the vector lanes are redundant. This constrains the sparsity to be relatively coarse grained, as irregularly scattered zero values are not beneficial.

\subsection{SparCE: Overview}

Figure 7 shows an overview of the micro-architectural and ISA enhancements proposed in SPARCE. We describe these extensions in detail, and demonstrate how they address the aforementioned challenges to leverage sparsity in DNNs.

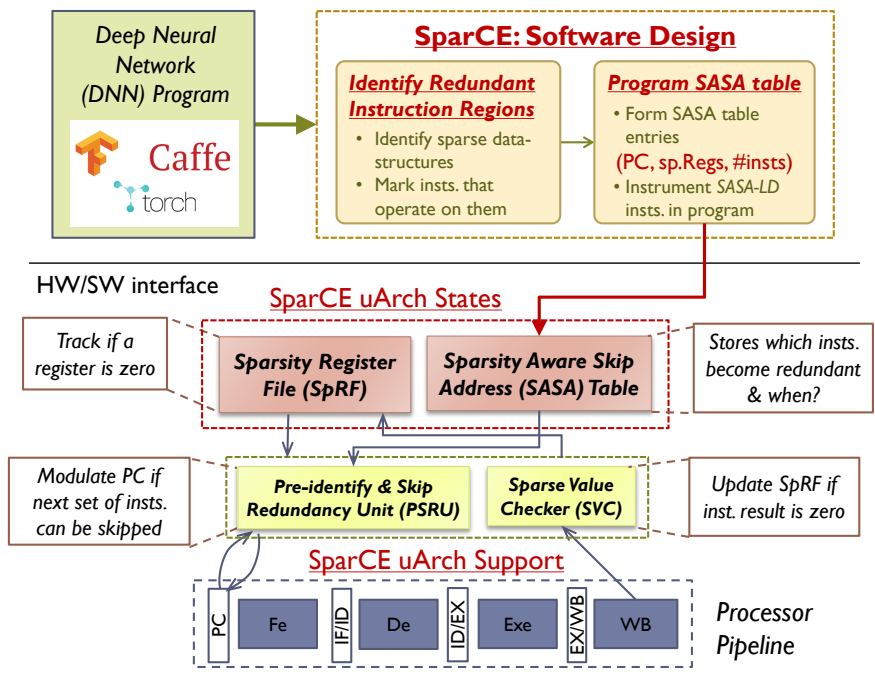

Fig. 7. SPARCE: Design Overview

\subsubsection{Micro-architectural states and ISA Extension}

In SPARCE, we augment the processor with two new microarchitectural states viz. Sparsity Register File (SpRF) and Sparsity Aware Skip Address (SASA) table. The SpRF is used to dynamically track which registers in the processor's register file contain zero values. The SpRF contains one entry (few bits) corresponding to each register in the register file. When an instruction that writes to a register retires, the SpRF is updated if the result is zero. The SASA table stores information about which instructions can be skipped and under what conditions. Specifically, each entry stores the program counter $(P C)$ of the instruction preceding a redundant instruction sequence, the index of the register that determines redundancy and the length of the sequence. Storing the $P C$ of the instruction preceding a redundant instruction sequence allows SPARCE to pre-identify whether the next set of instructions can be skipped and if so, skip them before the instructions are fetched.

SASA-LD Instruction. SPARCE enables software to explicitly identify potentially redundant instruction regions by pre-loading the SASA table at program startup, or before the program execution enters a given code region. To this end, we extend the ISA with a new instruction viz. $S A S A-L D$, which loads a given region of memory into the SASA table. As shown in Equation 2, the $S A S A-L D$ instruction takes a register operand $(R n)$ that points to the SASA table's location in memory and an immediate operand (size) that denotes the size of the SASA table.

$$
S A S A-L D \quad[R n], \# \text { size }
$$

At a given point in the program execution, the number of entries in the SASA table limits the number of redundant instruction regions that can be skipped by SPARCE. However, the SASA table can be periodically refreshed from memory as the program execution progresses. In the context of DNNs, we found that 20 entries in the SASA table suffice to capture all redundant instruction sequences, since the computational kernels are captured by a small number of library (e.g., BLAS) functions.

\subsubsection{Tracking, Pre-identifying, and Skipping Redundant In- structions}

SPARCE utilizes the SpRF and the SASA table to dynamically skip redundant instructions borne out of sparsity in the input data-structures. As shown in Figure 7, the micro-architecture of SPARCE is extended to support the following functions.

Track Sparse Registers. SPARCE contains a Sparse Value Checker $(S V C)$, which in the processor's writeback stage compares the result of each instruction to zero and if so, updates the entry corresponding to the instruction's destination register in the SpRF.

Pre-identify \& Skip Redundant Instructions. SPARCE is equipped with a Pre-identify and Skip Redundancy Unit (PSRU) that utilizes the SASA table to identify and skip redundant instruction regions. For each instruction, we check if its $P C$ contains an entry in the SASA table. An entry in the SASA table indicates that the instruction following the current instruction is the start of a potentially redundant instruction sequence. In this case, the PSRU checks the SpRF to identify if the registers indicated in the SASA table entry are currently zero. If so, it increments the $P C$ to the end of the redundant instruction sequence, thereby skipping instructions to benefit performance. If not, SPARCE proceeds to execute instructions in program order.

In summary, SPARCE uses the SpRF and the SASA table to seamlessly track sparse registers, pre-identify instruction sequences that are redundant and dynamically skip them before they are even fetched to improve performance.

\subsection{In-order SparCE Processor Pipeline}

We now explain how SPARCE can be integrated into an inorder processor. Figure 8 shows the block diagram of the overall SPARCE processor architecture. We start with a conventional 4stage (fetch, decode, execute/memory, and writeback) pipelined processor architecture implementing a RISC-style instruction set with at most 2 source register operands and one destination register operand. Although the SPARCE architecture is described in this section with a scalar execution unit for ease of illustration, it is directly applicable to vector processors with any number of SIMD execution lanes. We augment the processor with the following structures.

Sparsity Register File (SpRF). The SpRF is located at the fetch stage of the SPARCE processor. It is a multi-ported register file, with one entry corresponding to each register in the processor's register file. Each entry in the SpRF contains only two single-bit fields - isSparse and regUpdInFlight. The isSparse bit is set to 1 for registers containing zeros, and reset otherwise. The regUpdInFlight bit indicates whether an instruction modifying the register is in flight in any stage of the pipeline. For instance, an instruction modifying register $R d$, sets the $S p R F[R d][$ regUpdInFlight] field when it enters the decode stage and resets it after committing its result in the writeback stage. In determining whether a future 


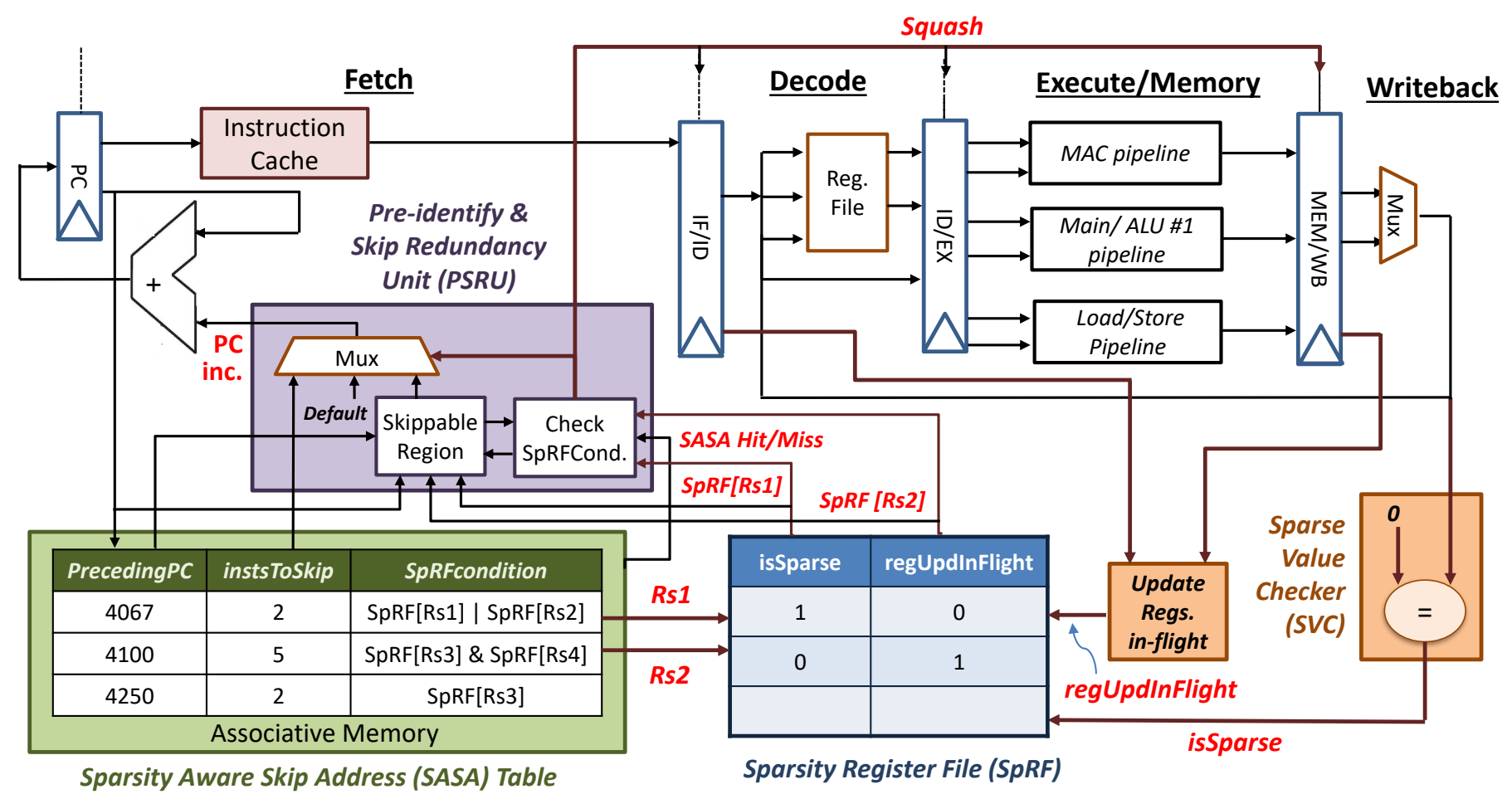

Fig. 8. Block diagram of SPARCE in-order processor architecture

instruction is redundant, the regUpdInFlight field ensures that we do not use a stale isSparse value when a more recent instruction updating this register is under execution.

Sparse Value Checker (SVC). The SVC is added to the writeback stage of the SPARCE processor. It contains a comparator that checks if the output of each instruction that updates a register is zero. It then correspondingly updates the SpRF. For example, when the output of an instruction with destination register $R d$ is zero, then $S p R F[R d][$ isSparse] is set and $S p R F[R d][r e g U p d I n F l i g h t]$ is reset.

Sparsity Aware Skip Address (SASA) table. The SASA table is also present in the fetch stage of the SPARCE processor. As shown in Figure 8 , the SASA table is an associative memory structure with three fields: (i) preceeding $P C$, which stores the $P C$ of the instruction prior to the redundant instruction sequence, (ii) SpRFCondition field which stores a Boolean combination of 2 register indices in the SpRF that should be satisfied for the instruction region to be skipped, and (iii) instsToSkip, which contains the length of the redundant instruction sequence. As an example, for the code in Figure 6 the SASA table entry to skip instructions 6-7 would be \{preceeding $P C=5$, SpRFCondition $=r 0 \mid r 1$, instsToSkip=2\}

Pre-identify and Skip Redundancy Unit (PSRU) The PSRU is also added to the fetch stage of SPARCE processor. The PSRU determines whether an instruction region specified in the SASA table can be skipped, and appropriately modulates the $P C$. Figure 9 shows the flowchart depicting the operation of PSRU. Given a $P C$, an associative lookup is performed on the preceeding $P C$ field of the SASA table. If the $P C$ is a hit in the SASA table, then the next instruction marks the beginning of a potentially redundant instruction sequence. To ascertain if the instruction sequence can be skipped, the PSRU reads the register indices specified in the SpRFCondition field of the SASA table (say $R_{s 1}$ and $R_{s 2}$ ) from the SpRF. If neither

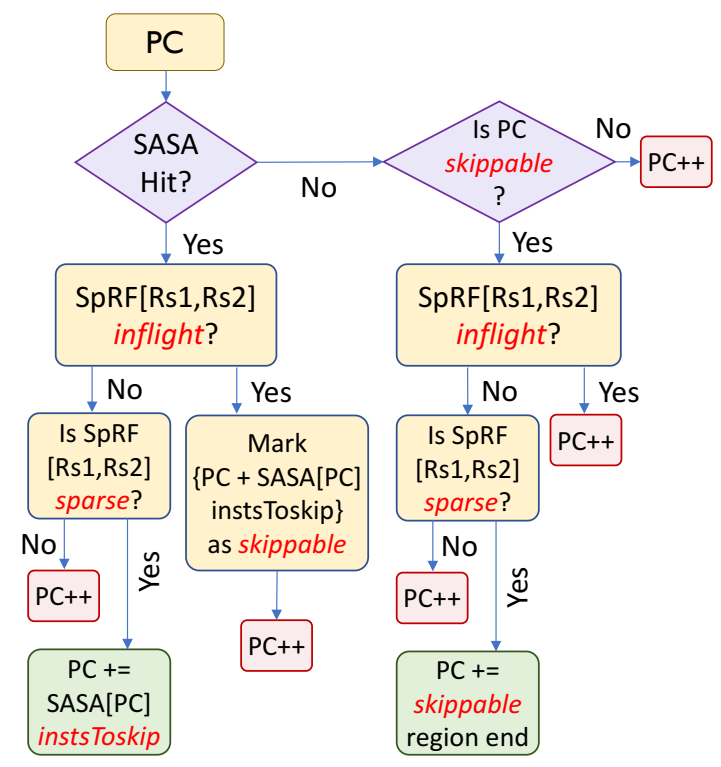

Fig. 9. Flowchart for pre-identifying and skipping redundancy

$S p R F\left[R_{s 1}\right]$ nor $\operatorname{SpRF}\left[R_{s 2}\right]$ have their regUpdInFlight field set, then PSRU computes the Boolean condition (specified in the $S p R F C$ ondition field) on their respective is Sparse fields. If the condition is satisfied, then the instruction region is deemed redundant and the $P C$ is incremented by instsToSkip to point to the instruction immediately following the end of the redundant region. If not, $P C$ is incremented by 1 and the instructions are executed in program order. However, if the regUpdInFlight field is set for either $\operatorname{SpRF}\left[R_{s 1}\right]$ or $\operatorname{SpRF}\left[R_{s 2}\right]$ and the Boolean condition in $S p R F C o n d i t i o n$ cannot be definitively evaluated, then the instruction region is temporarily marked as a skippable region within the PSRU. The $P C$ is incremented by 1 and the program execution is continued. It is worth noting that continuing 


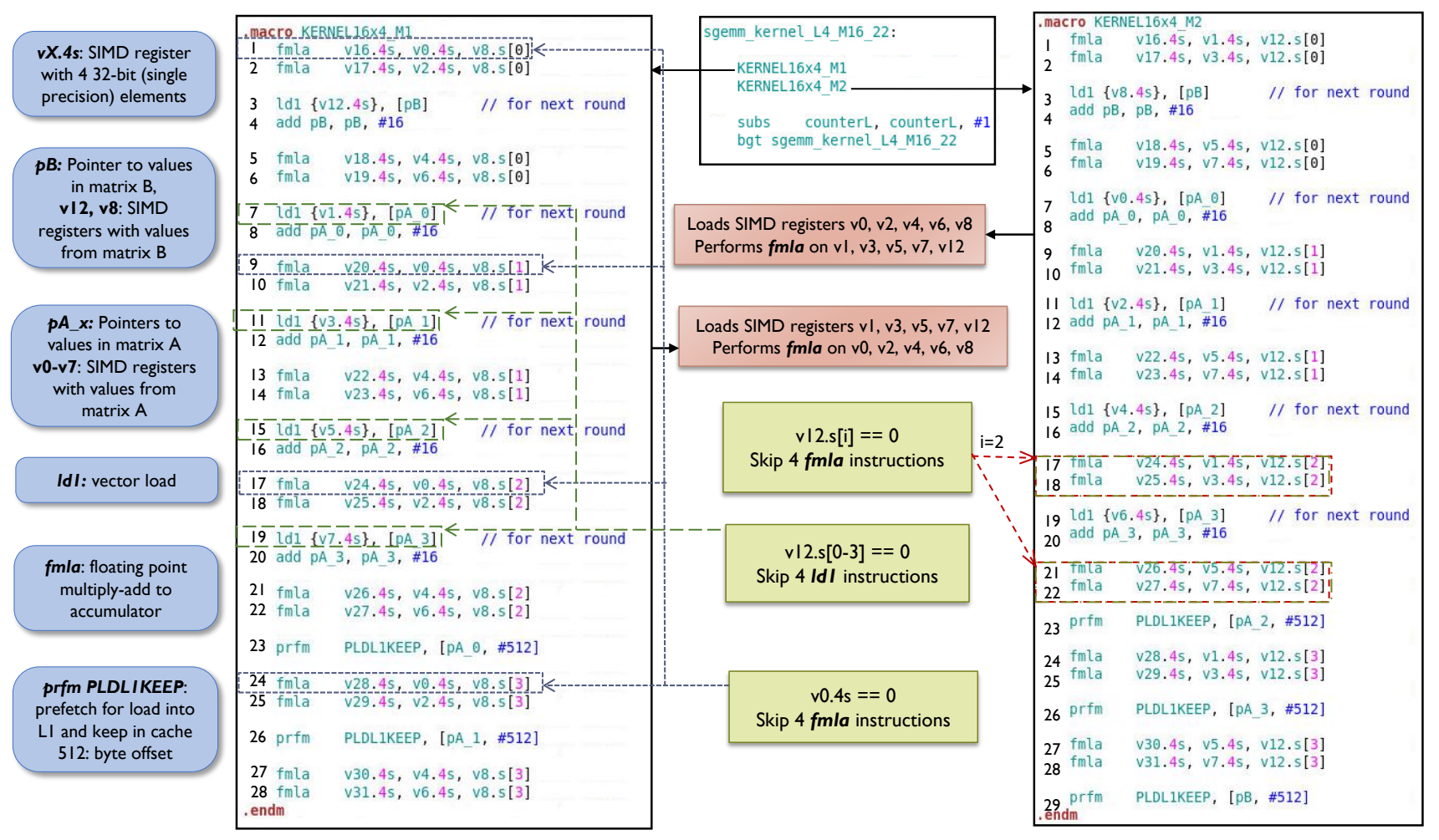

Fig. 10. Zero skipping for sgemm_kernel subroutine in BLAS

or aborting execution of a skippable region will not affect program functionality.

In the case when a $P C$ is not present in the SASA table, the PSRU checks if the $P C$ is part of an active skippable region. For the registers present in the SpRFCondition, the regUpdInFlight fields are re-checked from the SpRF. If they are reset, the Boolean condition in SpRFCondition is evaluated. If the skippable region is determined to be redundant, then the remaining instructions in the region are skipped by appropriately modifying the $P C$. Further, instructions belonging to the skippable region prior to the current $P C$ are squashed if they are still in flight. If the Boolean condition evaluates to a false, then the remaining instructions in the skippable region are executed. Finally, for a $P C$ that misses the SASA table and is not part of any active skippable region, the $P C$ is incremented by 1 to fetch the next instruction.

We note that the logic introduced in SPARCE to pre-identify redundant instructions executes in parallel with the instruction cache (ICache) access. The additional logic does not impact the latency of the fetch stage, as both the SASA table and the SpRF are significantly smaller structures compared to the ICache.

In summary, by using the SpRF and the SASA table, SPARCE dynamically tracks sparse registers, pre-identifies if an instruction region is redundant and skips instructions before they are even fetched to improve performance. Thus SPARCE enables DNN acceleration on GPPs by exploiting the sparsity resident in their data-structures.

\section{SOFTWARE FOR SPARCE Processsors}

To extract maximum performance from SPARCE, the software needs to suitably leverage the sparsity-aware micro-architectural extensions. In this section, we outline the key principles behind software design for SPARCE, and demonstrate them in the context of a highly optimized implementation of matrix multiplication (GEMM) from the OpenBLAS library.

\subsection{SparCE Software Design Steps}

The following steps need to be performed in software to leverage sparsity on SPARCE processors.

Identifying sparse data-structures and redundant instructions. One of the key requirements on software is to indicate (through the SASA table) which instruction regions are potentially redundant due to sparsity. To this end, sparse data-structures in the workload need to be first identified by the programmer. Next, when the application is compiled, the registers that hold the sparse datastructures and the instructions that load them from memory are identified. Then, a static dependency analysis of the instruction stream reveals the instructions that are affected by the sparse data-structures, which are then marked as potentially redundant instruction sequences. Following this, the condition for skipping each instruction sequence is derived from: which sparse datastructures affect the region, the registers containing them and the type of operation performed. Finally, the application assembly is instrumented with appropriate $S A S A-L D$ instructions.

Separate redundant instructions from instructions causing redundancy. To identify if an instruction region can be skipped, the instruction whose result makes them redundant should have completed execution. Therefore, in the context of in-order processors, they need to be spaced at least few instructions (3 in our as case as SVC is located in writeback stage) apart in the program, so that redundant instructions are not fetched and no pipeline bubble is introduced due to squashing redundant instructions. To this end, the instruction stream is re-ordered by inserting independent non-redundant instructions where needed to enable sufficient separation.

Mapping sparse data-structures on vector processors. In vector processors, typically one of the input operands is shared by all 
SIMD lanes, while the other is different across lanes. A vector instruction can be skipped only if computations performed on all SIMD lanes are redundant. Hence, it is better to map a sparse data-structure as the shared input operand, since the likelihood of all non-shared inputs being zero is low. If both data-structures are sparse, then the data-structure that exhibits the most block-wise sparsity is mapped as the non-shared input operand.

\subsection{Case Study: SparCE GEMM Routine}

Popular deep learning frameworks, such as Caffe [53], tensor flow [54], etc., execute DNNs as a series of matrix multiply operations, and leverage highly optimized software libraries such as BLAS (Basic Linear Algebra Subprograms) to realize them. Therefore, we develop a SPARCE version of the GEMM (Generalized Matrix Multiply) routine from the OpenBLAS library [55] targeting an ARM processor. We utilize the SPARCE-GEMM routine to quantify the reduction in DNN execution time achieved on a SPARCE processor.

Figure 10 shows the assembly program for the sgemm_kernel_l1_M16_22 subroutine, which performs singleprecision floating point matrix multiplication $(B \times A=C)$. The sgemm routine executes two smaller subroutines viz. kernel16x4_M1 (which we abbreviate as M1) and kernel16x4_M2 (M2) sequentially in a loop. The subroutines utilize vector registers $v 8$ and $v 12$ to hold the first input operand $(B)$ and registers $v 0-v 7$ to hold the second operand $(A)$. The intermediate results are computed in registers $v 16-v 31$. To optimize performance, each subroutine prefetches data for the other. For example, $M 1$ fetches data for operand $A$ into the odd registers, which are then used by $M 2$ in the subsequent iteration. The memory addresses are provided by scalar registers $p B$ and $p A \_0-p A \_3$. The subroutines also prefetch data in the L1-cache using the $p r f m$ instruction.

We identify redundant instruction sequences in the sgemm_kernel_l1_M16_22 subroutine assuming one of the input operands (say $B$ ) is sparse. The analysis can be easily extended to cover the scenario when either $A$ or both $A$ and $B$ are sparse. Since $B$ is sparse, it is beneficial to map it as the operand shared across the SIMD lanes, which is already the case in Figure 10 Even when one of the words in a vector register containing $B(v 8$ or $v 12$ ) is zero, $4 \mathrm{fmla}$ instructions can be skipped. For instance, when $v 12 . s[1]$ equals 0 , instructions $9,10,13,14$ in $M 2$ can be skipped. This forms 2 redundant instruction sequences (9-10 and 13-14), each of size 2. This amounts to 16 fmlas being skipped when the entire vector register ( $v 8$ or $v 12)$ is zero. It is worth noting that, had the sparse data-structure $(B)$ been mapped as the non-shared SIMD operand in the program, only 4 fmlas could be skipped even when the entire vector register is zero.

In addition to the fmla instructions being skipped, when the vector register is fully zero, the load instructions for the second operand can also be skipped. For example, when $v 12$ is zero, $l d 1$ instructions (7, 11, 15 and 19) for operand $A$ can be skipped in $M 1$. Also, note that we did not re-order any instruction in the sgemm routine, as the redundant instruction sequences were sufficiently spaced apart from the instruction that triggers their skipping. In the context of fmla instructions, the vector loads happen in a different subroutine owing to pre-fetching, and in the case of the $l d 1$ instructions, they were naturally spaced $>3$ instructions apart.

Based on the above analysis, Figure 11 shows the SASA table corresponding to the $M 1$ subroutine. We find a total of 12 entries

\begin{tabular}{|c|c|c|}
\hline PrecedingPC & instsToSkip & SpRFcondition \\
\hline $\begin{array}{c}\text { Bgt inst. in } \\
\text { sgemm }\end{array}$ & 2 & SpRF[v8[0]] \\
\hline $\mathrm{M} 1.4$ & 2 & SpRF[v8[0]] \\
\hline $\mathrm{M} 1.6$ & 1 & SpRF[v12] \\
\hline $\mathrm{M} 1.8$ & 2 & SpRF[v8[1]] \\
\hline $\mathrm{M} 1.10$ & 1 & SpRF[v12] \\
\hline $\mathrm{M} 1.12$ & 2 & SpRF[v8[1]] \\
\hline $\mathrm{M} 1.14$ & 1 & SpRF[v12] \\
\hline $\mathrm{M} 1.16$ & 2 & SpRF[v8[2]] \\
\hline $\mathrm{M} 1.18$ & 1 & SpRF[v12] \\
\hline $\mathrm{M} 1.20$ & 2 & SpRF[v8[2]] \\
\hline $\mathrm{M} 1.23$ & 2 & SpRF[v8[3]] \\
\hline $\mathrm{M} 1.26$ & 2 & SpRF[v8[3]] \\
\hline
\end{tabular}

\section{Fig. 11. SASA table entries for kernel16x4_M1 subroutine}

in the SASA table, 8 entries of size 2 for the 16 fmlas and 4 entries of size 1 for the $l d 1$ instructions.

SparCE in action. Figure 12 depicts the sequence of events that leads to instructions being skipped by SPARCE. First, when register $v 12$ is loaded by the $M 1$ subroutine, its SpRF entry is updated. Note that the isSparse field is a bit vector, one bit for each word in the vector register. Next, when instruction $M 2.12$ is fetched, it sees a hit in the SASA table and the reads $S p R F[12]$ to ascertain if the bit corresponding to $\operatorname{SpRF}[12][1]$ is sparse. Since that is the case, the $P C$ is incremented to directly fetch $M 2.15$.

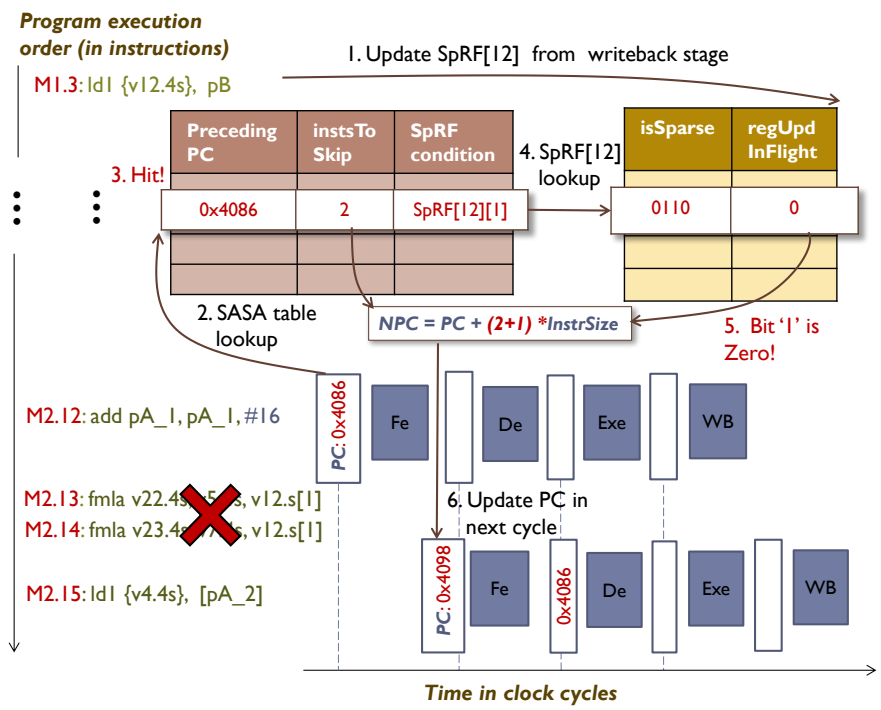

Fig. 12. SPARCE in action for sgemm routine

In summary, with minimal changes to software, SPARCE processors can leverage sparsity to improve performance.

\section{Experimental Methodology}

In this section, we present the methodology adopted in our experiments to evaluate SPARCE.

\subsection{Performance Evaluation}

We modeled the micro-architectural extensions proposed in SPARCE using the cycle-accurate gem5 architectural simulator [56]. The gem5 simulator was tightly integrated with the 
popular Caffe [53] deep learning framework, wherein the matrices corresponding to each layer and each input batch was formed in Caffe and fed into the gem5 simulator to perform the matrix computations. The results were fed back to Caffe to form the inputs for the next layer (or input batch), and so on. Figure 13 (a) shows the gem5 system configuration used in our experiments. All experiments were run in full-system mode. We evaluate SPARCE by measuring application level execution times under two scenarios. The first scenario targets embedded scalar processors that are present in ultra-low power edge/IoT devices and lack support for high performance libraries. We chose a scalar ARM v8 in-order processor architecture as the baseline. We leverage the modular nature of gem5 to cater to this scenario, wherein we disable support for advanced architectural features such as SIMD processing and pre-fetching in the ARM v8 processor. We then prototyped a direct convolution routine (which we call Dir-Conv-Scalar) that does not utilize the disabled features and used it in our experiments. The second scenario targets a reasonably sophisticated mobile processor for which we chose the ARM v8 in-order processor architecture with 4-way SIMD as the baseline. In this case, the matrix computations were realized using the highly-optimized OpenBLAS [55] based GEMM routines described in Section 4.2 We refer to this implementation as OpenBLAS-SIMD4.

\subsection{Power and Area Evaluation}

We implemented the hardware extensions of SPARCE at the Register Transfer Level (RTL) using Verilog HDL and synthesized them to IBM 45nm technology using Synopsys Design Compiler to measure its power and area overheads. For the configuration shown in Figure 13 (a), the area overhead is $0.4 \%$ of the ARM Cortex A35 core [43]. Thus the area overhead of SPARCE is quite minimal allowing its deployment in the resource-constrained embedded platforms.

\begin{tabular}{|l|l|}
\hline $\begin{array}{l}\text { Processor } \\
\text { config. }\end{array}$ & ARMv8-A, In-order \\
\hline $\begin{array}{l}\text { SPARCE } \\
\text { config. }\end{array}$ & $\begin{array}{l}\text { 20 SASA table entries, 32 } \\
\text { SpRF entries }\end{array}$ \\
\hline L1 Cache & $\begin{array}{l}\text { Split I\&D, 32KB I cache, } \\
\text { 64KB D cache, 2-way set } \\
\text { associative I \& D cache, } \\
\text { 64B line, 3-cycles/access }\end{array}$ \\
\hline L2 Cache & $\begin{array}{l}\text { Unified 2MB 8-way set } \\
\text { associative, 64B line, 12 } \\
\text { cycles/ access }\end{array}$ \\
\hline
\end{tabular}

(a)

Fig. 13. (a) Gem5 simulation parameters (b) Application benchmarks

\subsection{Benchmarks}

Our benchmark suite, listed in Figure 13 b), consists of 6 state-ofthe-art image-recognition DNNs viz. CIFAR-Caffe DNN using the CIFAR-10 dataset [57], and AlexNet [52], VGG-16 [2], ResNet50 [1], GoogleNet [3] and Deep Compression-AlexNet [36] using the ImageNet dataset. These benchmarks contained 5-50 layers and took 0.01-15.4 Billion scalar operations to classify an image. We utilized pre-trained models from the Caffe Model Zoo to evaluate SPARCE in the context of inference. For training, we utilized only the smaller CIFAR-10 benchmark, as training ImageNet models on gem5 was prohibitively time consuming.
It is noteworthy that all benchmarks exhibited dynamic sparsity in features and errors, while only Deep Compression-AlexNet exhibited static parsity in weights.

\section{Results}

In this section, we present the results of our experiments that highlight the advantages of SPARCE.

\subsection{Performance and Energy Improvement}

Figure 14 shows the normalized execution time benefits of SPARCE over the baseline processor for both inference and training. In the context of Dir-Conv-Scalar, the reduction in application runtime ranges between 19\%-31\% across the benchmarks. In contrast, OpenBLAS-SIMD4 demonstrates benefits in the range of $8 \%-15 \%$ reduction in runtime. This is because $f m l a$ instructions occupy a much smaller fraction of their runtime, as their execution engines are more sophisticated - multiple SIMD lanes, low floating point instruction latency etc. Also, since they support features such as prefetching where the data is already fetched into the higher levels of the memory subsystem, avoiding redundant data fetches has a less prominent impact on performance.

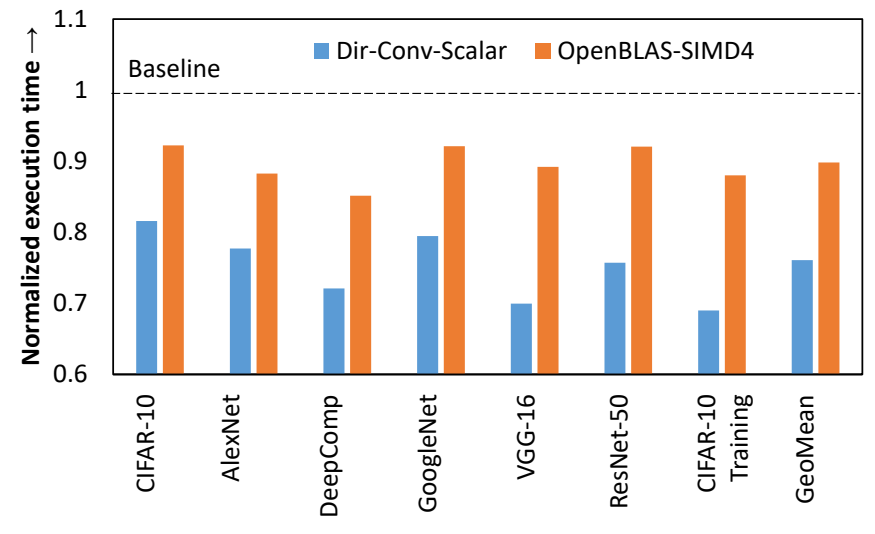

Fig. 14. Improvement in execution time at the application level

Among the benchmarks, the execution time benefits are largely proportional to the amount of sparsity that they exhibit (Figure 2 in Section 22. The Deep Compression-AlexNet benchmark achieves the most benefits because both its feature and weight datastructures are sparse, as opposed to other benchmarks whose weight data-structure is dense. In the context of training, the backpropagation step achieves more improvement compared to forward propagation. This stems from the fact that the error datastructure is more sparse compared to features.

Execution Time Breakdown. To better appreciate the improvements achieved by SPARCE, Figure 15 hierarchically breaks down the application execution time (in the context of both Dir-ConvScalar and OpenBlas-SIMD4 implementations) into components that can and cannot be impacted by SPARCE. At the top level, the solid yellow and gray colors represent the execution time fraction that cannot be improved by SPARCE. This is primarily constituted by auxiliary DNN operations such as activation functions, subsampling and others, which represent $1.9 \%$ and $12.2 \%$ of the runtime for Dir-Conv-Scalar and OpenBlas-SIMD4 implementations, respectively. It is noteworthy that although these 
operations occupy $<1 \%$ of the total DNN FLOPs, they occupy a substantially larger fraction of the runtime for the OpenBLASSIMD4 implementation. This is owed to the fact that they are typically memory-bound (higher Bytes/FLOP ratio), which is further amplified as matrix multiply operations are significantly optimized by the GEMM subroutine. Also, since the inputs to the DNN are typically dense, the first DNN layer exhibits little redundancy. This occupies $14.3 \%$ and $16.9 \%$ of the total runtimes of AlexNet for Dir-Conv-Scalar and OpenBLAS-SIMD4 implementations, respectively. The fraction grows smaller in deeper networks such as ResNet and VGG.

In Figure 15 the green color bars denote the computations that can accelerated by leveraging sparsity $(\sim 71 \%)$. For DirConv-Scalar implementation, this is limited to $83.6 \%$ of the baseline AlexNet runtime. Since AlexNet contains $\sim 36 \%$ redundant computations (Figure 4 in Section 2), the best case benefits are limited to $29.8 \%$, of which SPARCE achieves $22.3 \%$. For the OpenBLAS-SIMD4 implementation, the underlying GEMM involves supplementary operations like memory allocate, copy and free operations, which as marked by the dotted patterns and consumes $27 \%$ of the total execution time. This constraints the opportunity for SPARCE to $\sim 44 \%$ of AlexNet runtime as shown by the diagonally hatched portions in Figure 15 Since AlexNet contains $\sim 36 \%$ redundant computations (Figure 4 in Section 2, the best case benefits are limited to $\sim 16 \%$, of which SPARCE achieves $12 \%$ improvement as other control operations such as pointer arithmetic, prefetching etc. present within the loop body cannot be avoided.

\section{$\square$ Auxiliary Computations \\ $\square$ Other Layers \\ $\square$ First DNN Layer \\ $₫$ Other Layers - sgemm Compute}

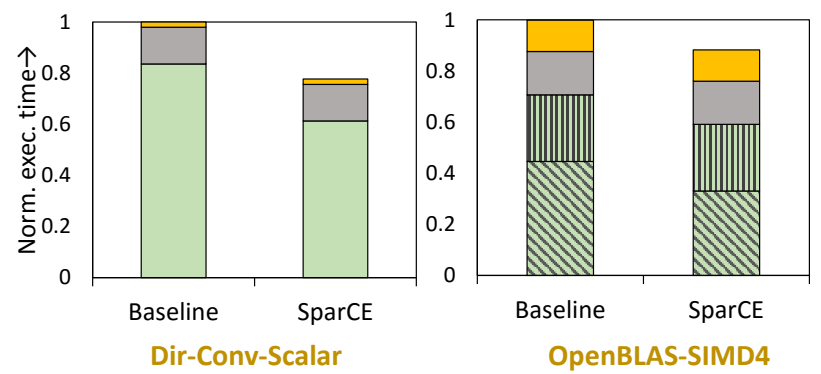

Fig. 15. Execution time breakdown for AlexNet

Layer-wise Benefits. We now present the layer-wise breakdown of the benefits quantified in terms of the execution time, instructions and data cache (D-Cache) accesses skipped for the convolutional layers of AlexNet. Figure 16 shows the benefits in the context of both SIMD and scalar processor implementations. As shown in Figure 16 , we are able to achieve, on an average, $39.4 \%$ reduction in instruction count and $35.1 \%$ reduction in D-Cache accesses for Dir-Conv implementations on low-power embedded scalar processors. The reduction in instruction count and D-Cache accesses amount to $30.5 \%$ and $14 \%$ respectively for OpenBLAS-SIMD4 implementations. We also observe the benefits are typically larger for layers deeper in the DNN, as they typically exhibit more sparsity.

Energy Benefits. Power evaluation of the SPARCE reveals that it consumes $1.74 \mathrm{~mW}$ at $1 \mathrm{GHz}$, which amounts to $1.9 \%$ of the $90 \mathrm{~mW}$ power consumed by even the most power-efficient
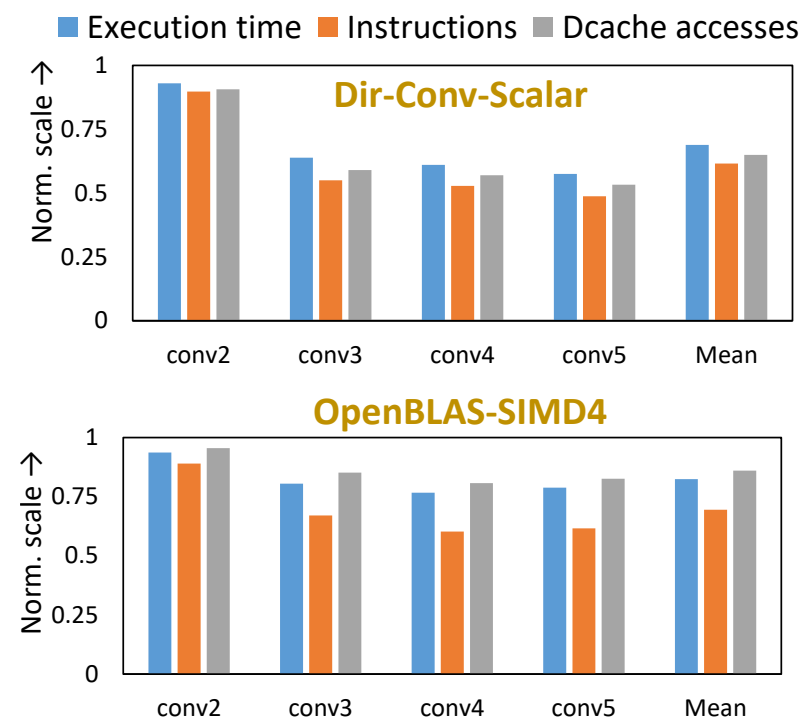

Fig. 16. Layer-wise benefits breakdown for AlexNet

baseline ARM v8 processor, the Cortex A35 processor [43]. Accordingly, the execution time benefits translate to benefits in the range of $16.9 \%-28.7 \%$ reduction in application-level energy for a Dir-Conv-Scalar implementation. In the context of OpenBLASSIMD4 implementations, the reduction in energy ranges between $6.1 \%-13.2 \%$ across the benchmarks.

\subsection{Performance Scaling with Sparsity}

We now study how the performance of SPARCE scales with increasing levels of sparsity. To this end, we considered a matrix multiplication problem $(B \times A=C)$, wherein the dimensions of the input matrices $B$ and $A$ were $169 \times 3456$ and $3456 \times 384$ respectively. We varied the sparsity of the $B$ matrix by constraining the number of zero entries. The location of the zeros and other entries of the matrices were chosen at random. Figure 17 shows how the execution time and the fraction of instructions executed varies with sparsity in the context of both Dir-Conv-Scalar and OpenBLASSIMD4 implementations. We find that both implementations exhibit strong performance scaling with sparsity, outlining the ability of SPARCE to efficiently skip computations. We find the number of instructions executed to be larger than ideal (dotted line in Figure 17) due to the presence of control instructions for pointer arithmetic, loop counts etc., in the program, which cannot be skipped. Also, the disparity in the fraction of instructions executed and the resultant execution time benefits is more pronounced for the OpenBLAS-SIMD4 implementation. We attribute this to the intelligent instruction ordering in the GEMM routine utilized in the implementation, wherein computations are aggressively overlapped with data-fetches. Therefore, even if computations are skipped, the improvement in performance is limited by the time taken for the data-fetches.

\subsection{Operand Ordering in SparCE OpenBLAS-SIMD4 Im- plementations}

As described in Section 4 based on the amount of sparsity exhibited by the data-structures, mapping the right datastructure as the shared-SIMD operand can have a considerable impact on performance. In the case of all benchmarks other than Deep Compression-AlexNet, only the feature 


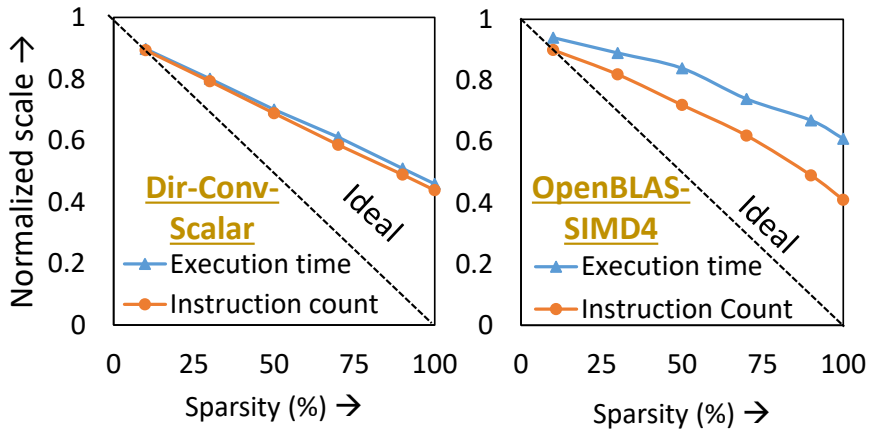

Fig. 17. SPARCE performance scaling with sparsity

data-structure is sparse. Therefore, mapping it as the sharedSIMD operand (Matrix $B$ in the sgemm subroutine) would yield the best benefits. Figure 18 shows the performance improvement achieved when operands are ordered in both ways viz. Features $\times$ Weights and Weights $\times$ Features. In the context of AlexNet, we find that mapping features as the sharedSIMD operand yields $1.86 \times$ better benefits $(\sim 12 \%$ vs. $6.5 \%)$ compared to mapping the non-sparse weight data-structure. For the Deep CompressionAlexNet network, since both feature and weight datastructures are sparse, the disparity in performance due to operand ordering is relatively small $(<2 \%)$. Even in this case, we find that choosing features as the shared-SIMD operand is beneficial. This is attributed to the fact that some of the weight matrices have high degree of sparsity, and their zero entries are typically clustered. Therefore, using weights as the non-shared

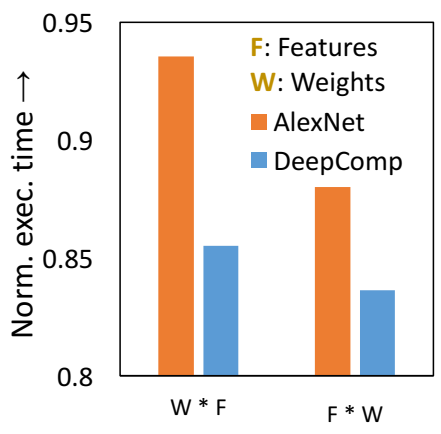

Fig. 18. Impact of operand ordering on performance

SIMD operand has less of an adverse impact on performance compared to using features.

\section{Related Work}

Prior research efforts that target improving the computational efficiency of DNNs can be grouped into the following broad classes.

Software parallelization on multi-cores and GPUs. A large number of previous efforts have been directed towards developing techniques for efficient parallelization of DNNs on multi-core servers and GPUs [13]-[19]. However, the scalability of these techniques is often limited by synchronization and communication bottlenecks.

Specialized accelerators. Developing specialized hardware architectures has been an attractive approach to improve the computational efficiency of DNNs. These accelerators [20]-[26], [28][31] utilize specialized processing cores, interconnect network and other hardware-software co-design methodologies to leverage the different forms of compute and data reuse patterns in DNNs.

Approximate computing. DNNs and the applications that use them are intrinsically resilient to errors in their underlying compu- tations. Approximate computing techniques, such as low-precision implementations [32]-[35], model compression [36], [37] and others [38], [39], leverage this property to improve the computational efficiency of DNNs.

Post-CMOS technology. Post-CMOS technologies such as memristors and spintronics have succeeded in realizing the computational primitives of DNNs through their intrinsic device characteristics. Implementations of small scale DNNs using memristor crossbar arrays [40], [42] and spintronic devices [41] have demonstrated significant promise.

All the above efforts are complementary to SPARCE, as unlike above efforts, we attempt to leverage sparsity in the different DNN data-structures to improve efficiency on cost constrained embedded platforms comprising of mainly a GPP core.

Exploiting sparsity. Prior efforts that exploit sparsity to improve DNN efficiency can be grouped to two classes based on whether they exploit static sparsity or dynamic sparsity, as shown in Figure 1. Specialized sparse architectures that are capable of exploiting both static and dynamic sparsity incorporate a variety of compression techniques and zero-skipping schemes to reduce storage requirement and avoid redundant multiplications in accelerators [31], [44]-[48].

On the other hand, software approaches that exploit weight sparsity on GPPs take advantage of sparse matrix libraries. These sparse libraries usually yield performance improvement only under extreme levels of sparsity ( $>95 \%)$. Since DNNs naturally exhibit sparsity in the range of $40 \%-70 \%$, a few research efforts force more weight sparsity into DNNs using sparse decomposition methods, etc. [49], [50] or customize the pruning to match the underlying hardware organization [51]. These invariably come at the cost of training overhead or loss of functional accuracy. In contrast, we propose micro-architectural extensions to GPPs that can exploit both dynamic and static sparsity while being effective even under intermediate levels. Thus, SPARCE is able to exploit feature sparsity in several state-of-the-art dense DNN models as well as both feature and weight sparsity in existing pruned DNN models.

\section{Conclusion}

As DNNs pervade the spectrum of computing devices, new approaches to improve their computational efficiency on resourceconstrained IoT/ edge devices becomes critical. In this work, we accelerate DNNs on GPPs, which are an indispensable part of IoT/ edge devices, by exploiting sparsity in the different DNN data-structures. To this end, we propose sparsity aware general purpose core extensions (SPARCE) that enable GPPs to efficiently leverage sparsity, while being minimally intrusive and low-overhead. SPARCE comprises of two key micro-architectural enhancements. First, a Sparsity Register File (SpRF) dynamically tracks zero-valued registers in the processor. Next, a Sparsity Aware Skip Address (SASA) table indicates potentially redundant instruction sequences and the conditions under which they can be skipped. A Pre-identify and Skip Redundancy Unit (PSRU) combines the information from the SpRF and the SASA table to dynamically pre-identify if an instruction sequence can be skipped, and if so masks it from being fetched and executed. We evaluate SPARCE on 6 image-recognition DNNs in the context of both training and inference. Our evaluations reveal that SPARCE is a promising design that allows us to exploit all forms of static and dynamic sparsity to accelerate DNNs on GPPs. 


\section{REFERENCES}

[1] Kaiming He, Xiangyu Zhang, Shaoqing Ren, and Jian Sun. Deep residual learning for image recognition. CoRR, abs/1512.03385, 2015.

[2] K. Simonyan and A. Zisserman. Very deep convolutional networks for large-scale image recognition. CoRR, abs/1409.1556, 2014.

[3] Christian Szegedy, Wei Liu, Yangqing Jia, Pierre Sermanet, Scott E. Reed, Dragomir Anguelov, Dumitru Erhan, Vincent Vanhoucke, and Andrew Rabinovich. Going deeper with convolutions. CoRR, abs/1409.4842, 2014.

[4] S. Venugopalan, M. Rohrbach, J. Donahue, R. J. Mooney, T. Darrell, and K. Saenko. Sequence to sequence - video to text. In 2015 IEEE International Conference on Computer Vision, ICCV 2015, Santiago, Chile, December 7-13, 2015, pages 4534-4542, 2015.

[5] Awni Y. Hannun, Carl Case, Jared Casper, Bryan Catanzaro, Greg Diamos, Erich Elsen, Ryan Prenger, Sanjeev Satheesh, Shubho Sengupta, Adam Coates, and Andrew Y. Ng. Deep speech: Scaling up end-to-end speech recognition. CoRR, abs/1412.5567, 2014.

[6] Xiang Zhang and Yann LeCun. Text understanding from scratch. CoRR, $\mathrm{abs} / 1502.01710,2015$.

[7] Geoffrey Hinton, Li Deng, Dong Yu, George Dahl, Abdel rahman Mohamed, Navdeep Jaitly, Andrew Senior, Vincent Vanhoucke, Patrick Nguyen, Tara Sainath, and Brian Kingsbury. Deep neural networks for acoustic modeling in speech recognition. Signal Processing Magazine, 2012.

[8] Jiwei Li, Michel Galley, Chris Brockett, Georgios P. Spithourakis, Jianfeng Gao, and Bill Dolan. A persona-based neural conversation model. March 2016.

[9] Improving photo search: A step across the semantic gap. Google Research blog, 2013.

[10] Y. Taigman, M. Yang, M. Ranzato, and L. Wolf. Deepface: Closing the gap to human-level performance in face verification. In 2014 IEEE Conference on Computer Vision and Pattern Recognition, pages 17011708, June 2014.

[11] Introducing deeptext: Facebook's text understanding engine: https://code.facebook.com/posts/181565595577955/introducingdeeptext-facebook-s-text-understanding-engine/. Facebook Code, 2016.

[12] Apple is turning Siri into a next-level Artificial Intelligence: http://mashable.com/2016/06/13/siri-sirikit-wwdc2016analysis/hLMSxZKVnEqO. Mashable, 2016.

[13] Alex Krizhevsky. One weird trick for parallelizing convolutional neural networks. CoRR, abs/1404.5997, 2014.

[14] Forrest N. Iandola, Khalid Ashraf, Matthew W. Moskewicz, and Kurt Keutzer. Firecaffe: near-linear acceleration of deep neural network training on compute clusters. CoRR, abs/1511.00175, 2015.

[15] Jeffrey Dean, Greg Corrado, Rajat Monga, Kai Chen, Matthieu Devin, Mark Mao, Marc'aurelio Ranzato, Andrew Senior, Paul Tucker, Ke Yang, Quoc V. Le, and Andrew Y. Ng. Large scale distributed deep networks. In P. Bartlett, F.c.n. Pereira, C.j.c. Burges, L. Bottou, and K.q. Weinberger, editors, Advances in Neural Information Processing Systems 25, pages 1232-1240. 2012.

[16] M. Rhu, N. Gimelshein, J. Clemons, A. Zulfiqar, and S. W. Keckler. vdnn: Virtualized deep neural networks for scalable, memory-efficient neural network design. In 2016 49th Annual IEEE/ACM International Symposium on Microarchitecture (MICRO), pages 1-13, Oct 2016.

[17] Dipankar Das, Sasikanth Avancha, Dheevatsa Mudigere, Karthikeyan Vaidyanathan, Srinivas Sridharan, Dhiraj D. Kalamkar, Bharat Kaul, and Pradeep Dubey. Distributed deep learning using synchronous stochastic gradient descent. CoRR, abs/1602.06709, 2016.

[18] Aleksandar Zlateski, Kisuk Lee, and H. Sebastian Seung. ZNN - A fast and scalable algorithm for training $3 \mathrm{~d}$ convolutional networks on multi-core and many-core shared memory machines. In 2016 IEEE International Parallel and Distributed Processing Symposium, IPDPS 2016, Chicago, IL, USA, May 23-27, 2016, pages 801-811, 2016.

[19] Neon, nervana systems: http://neon.nervanasys.com/docs/latest/index.html. 2016.

[20] C. Farabet, B. Martini, B. Corda, P. Akselrod, E. Culurciello, and Y. LeCun. Neuflow: A runtime reconfigurable dataflow processor for vision. In CVPR 2011 WORKSHOPS, pages 109-116, June 2011.

[21] Srimat Chakradhar, Murugan Sankaradas, Venkata Jakkula, and Srihari Cadambi. A dynamically configurable coprocessor for convolutional neural networks. SIGARCH Comput. Archit. News, 38(3):247-257, June 2010.

[22] Tianshi Chen, Zidong Du, Ninghui Sun, Jia Wang, Chengyong Wu, Yunji Chen, and Olivier Temam. Diannao: A small-footprint high-throughput accelerator for ubiquitous machine-learning. In Proceedings of the 19th International Conference on Architectural Support for Programming
Languages and Operating Systems, ASPLOS '14, pages 269-284, New York, NY, USA, 2014. ACM.

[23] Yunji Chen, Tao Luo, Shaoli Liu, Shijin Zhang, Liqiang He, Jia Wang, Ling Li, Tianshi Chen, Zhiwei Xu, Ninghui Sun, and Olivier Temam. Dadiannao: A machine-learning supercomputer. In Proceedings of the 47th Annual IEEE/ACM International Symposium on Microarchitecture, MICRO-47, pages 609-622, Washington, DC, USA, 2014. IEEE Computer Society.

[24] Swagath Venkataramani, Ashish Ranjan, Subarno Banerjee, Dipankar Das, Sasikanth Avancha, Ashok Jagannathan, Ajaya Durg, Dheemanth Nagaraj, Bharat Kaul, Pradeep Dubey, and Anand Raghunathan. Scaledeep: A scalable compute architecture for learning and evaluating deep networks. In Proceedings of the 44th Annual International Symposium on Computer Architecture, ISCA '17, pages 13-26, New York, NY, USA, 2017. ACM.

[25] N. Jouppi, C. Young, N. Patil, D. Patterson, G. Agrawal, R. Bajwa, S. Bates, S. Bhatia, N. Boden, A. Borchers, R. Boyle, P. Cantin, C. Chao, C. Clark, J. Coriell, M. Daley, M. Dau, J. Dean, B. Gelb, T. V. Ghaemmaghami, R. Gottipati, W. Gulland, R. Hagmann, C. R. Ho, D. Hogberg, J. Hu, R. Hundt, D. Hurt, J. Ibarz, A. Jaffey, A. Jaworski, A. Kaplan, H. Khaitan, A. Koch, N. Kumar, S. Lacy, J. Laudon, J. Law, D. Le, C. Leary, Z. Liu, K. Lucke, A. Lundin, G. MacKean, A. Maggiore, M. Mahony, K. Miller, R. Nagarajan, R. Narayanaswami, R. Ni, K. Nix, T. Norrie, M. Omernick, N. Penukonda, A. Phelps, J. Ross, M. Ross, A. Salek, E. Samadiani, C. Severn, G. Sizikov, M. Snelham, J. Souter, D. Steinberg, A. Swing, M. Tan, G. Thorson, B. Tian, H. Toma, E. Tuttle, V. Vasudevan, R. Walter, W. Wang, E. Wilcox, and D. H. Yoon. In-datacenter performance analysis of a tensor processing unit. In Proceedings of the 44th Annual International Symposium on Computer Architecture, ISCA '17, pages 1-12, New York, NY, USA, 2017. ACM.

[26] Brandon Reagen, Paul Whatmough, Robert Adolf, Saketh Rama, Hyunkwang Lee, Sae Kyu Lee, José Miguel Hernández-Lobato, GuYeon Wei, and David Brooks. Minerva: Enabling low-power, highlyaccurate deep neural network accelerators. In Proceedings of the 43rd International Symposium on Computer Architecture, ISCA '16, pages 267-278, Piscataway, NJ, USA, 2016. IEEE Press.

[27] Swagath Venkataramani, Vinay K. Chippa, Srimat T. Chakradhar, Kaushik Roy, and Anand Raghunathan. Quality programmable vector processors for approximate computing. In Proceedings of the 46th Annual IEEE/ACM International Symposium on Microarchitecture, MICRO-46, pages 1-12, New York, NY, USA, 2013. ACM.

[28] S. Eldridge, A. Waterland, M. Seltzer, J. Appavoo, and A. Joshi. Towards general-purpose neural network computing. In 2015 International Conference on Parallel Architecture and Compilation (PACT), pages 99-112, Oct 2015.

[29] Abhinandan Majumdar, Srihari Cadambi, Michela Becchi, Srimat T. Chakradhar, and Hans Peter Graf. A massively parallel, energy efficient programmable accelerator for learning and classification. ACM Trans. Archit. Code Optim., 9(1):6:1-6:30, March 2012.

[30] V. Gokhale, J. Jin, A. Dundar, B. Martini, and E. Culurciello. A 240 g-ops/s mobile coprocessor for deep neural networks. In 2014 IEEE Conference on Computer Vision and Pattern Recognition Workshops, pages 696-701, June 2014.

[31] Y. H. Chen, T. Krishna, J. S. Emer, and V. Sze. Eyeriss: An energyefficient reconfigurable accelerator for deep convolutional neural networks. IEEE Journal of Solid-State Circuits, 52(1):127-138, Jan 2017.

[32] Suyog Gupta, Ankur Agrawal, Kailash Gopalakrishnan, and Pritish Narayanan. Deep learning with limited numerical precision. In Proceedings of the 32Nd International Conference on International Conference on Machine Learning - Volume 37, ICML'15, pages 1737-1746. JMLR.org, 2015.

[33] Swagath Venkataramani, Ashish Ranjan, Kaushik Roy, and Anand Raghunathan. Axnn: Energy-efficient neuromorphic systems using approximate computing. In Proceedings of the 2014 International Symposium on Low Power Electronics and Design, ISLPED '14, pages 27-32, New York, NY, USA, 2014. ACM.

[34] Chenzhuo Zhu, Song Han, Huizi Mao, and William J. Dally. Trained ternary quantization. CoRR, abs/1612.01064, 2016.

[35] Matthieu Courbariaux, Yoshua Bengio, and Jean-Pierre David. Binaryconnect: Training deep neural networks with binary weights during propagations. CoRR, abs/1511.00363, 2015.

[36] Song Han, Huizi Mao, and William J. Dally. Deep compression: Compressing deep neural network with pruning, trained quantization and huffman coding. CoRR, abs/1510.00149, 2015.

[37] Max Jaderberg, Andrea Vedaldi, and Andrew Zisserman. Speeding up convolutional neural networks with low rank expansions. CoRR, abs/1405.3866, 2014. 
[38] Frank Seide, Hao Fu, Jasha Droppo, Gang Li, and Dong Yu. 1-bit stochastic gradient descent and application to data-parallel distributed training of speech dnns. In Interspeech 2014, September 2014.

[39] Wei Zhang, Suyog Gupta, Xiangru Lian, and Ji Liu. Staleness-aware async-sgd for distributed deep learning. CoRR, abs/1511.05950, 2015.

[40] Xiaoxiao Liu, Mengjie Mao, Beiye Liu, Hai Li, Yiran Chen, Boxun Li, Yu Wang, Hao Jiang, Mark Barnell, Qing Wu, and Jianhua Yang. Reno: A high-efficient reconfigurable neuromorphic computing accelerator design. In Proceedings of the 52Nd Annual Design Automation Conference, DAC '15, pages 66:1-66:6, New York, NY, USA, 2015. ACM.

[41] S. G. Ramasubramanian, R. Venkatesan, M. Sharad, K. Roy, and A. Raghunathan. Spindle: Spintronic deep learning engine for largescale neuromorphic computing. In Low Power Electronics and Design (ISLPED), 2014 IEEE/ACM International Symposium on, pages 15-20, Aug 2014.

[42] A. Shafiee, A. Nag, N. Muralimanohar, R. Balasubramonian, J. P. Strachan, M. Hu, R. S. Williams, and V. Srikumar. Isaac: A convolutional neural network accelerator with in-situ analog arithmetic in crossbars. In 2016 ACM/IEEE 43rd Annual International Symposium on Computer Architecture (ISCA), pages 14-26, June 2016.

[43] ARM Announces New Cortex-A35 CPU - Ultra-High Efficiency For Wearables \& More: https://www.anandtech.com/show/9769/armannounces-cortex-a35. Anandtech, 2015.

[44] J. Albericio, P. Judd, T. Hetherington, T. Aamodt, N. E. Jerger, and A. Moshovos. Cnvlutin: Ineffectual-neuron-free deep neural network computing. In 2016 ACM/IEEE 43rd Annual International Symposium on Computer Architecture (ISCA), pages 1-13, June 2016.

[45] Song Han, Xingyu Liu, Huizi Mao, Jing Pu, Ardavan Pedram, Mark A. Horowitz, and William J. Dally. EIE: efficient inference engine on compressed deep neural network. CoRR, abs/1602.01528, 2016.

[46] Angshuman Parashar, Minsoo Rhu, Anurag Mukkara, Antonio Puglielli, Rangharajan Venkatesan, Brucek Khailany, Joel Emer, Stephen W. Keckler, and William J. Dally. Scnn: An accelerator for compressed-sparse convolutional neural networks. In Proceedings of the 44th Annual International Symposium on Computer Architecture, ISCA '17, pages 27-40, New York, NY, USA, 2017. ACM.

[47] S. Zhang, Z. Du, L. Zhang, H. Lan, S. Liu, L. Li, Q. Guo, T. Chen, and Y. Chen. Cambricon-X: An accelerator for sparse neural networks. In 2016 49th Annual IEEE/ACM International Symposium on Microarchitecture (MICRO), pages 1-12, Oct 2016.

[48] Hyunsun Park, Dongyoung Kim, Junwhan Ahn, and Sungjoo Yoo. Zero and data reuse-aware fast convolution for deep neural networks on gpu. In Proceedings of the Eleventh IEEE/ACM/IFIP International Conference on Hardware/Software Codesign and System Synthesis, CODES '16, pages 33:1-33:10, New York, NY, USA, 2016. ACM.

[49] Baoyuan Liu, Min Wang, H. Foroosh, M. Tappen, and M. Penksy. Sparse convolutional neural networks. In 2015 IEEE Conference on Computer Vision and Pattern Recognition (CVPR), pages 806-814, June 2015.

[50] Wei Wen, Chunpeng Wu, Yandan Wang, Yiran Chen, and Hai Li. Learning structured sparsity in deep neural networks. In D. D. Lee, M. Sugiyama, U. V. Luxburg, I. Guyon, and R. Garnett, editors, Advances in Neural Information Processing Systems 29, pages 2074-2082. Curran Associates, Inc., 2016.

[51] Jiecao Yu, Andrew Lukefahr, David Palframan, Ganesh Dasika, Reetuparna Das, and Scott Mahlke. Scalpel: Customizing dnn pruning to the underlying hardware parallelism. In Proceedings of the 44th Annual International Symposium on Computer Architecture, ISCA '17, pages 548-560, New York, NY, USA, 2017. ACM.

[52] Alex Krizhevsky, Ilya Sutskever, and Geoffrey E Hinton. Imagenet classification with deep convolutional neural networks. In F. Pereira, C. J. C. Burges, L. Bottou, and K. Q. Weinberger, editors, Advances in Neural Information Processing Systems 25, pages 1097-1105. Curran Associates, Inc., 2012.

[53] Yangqing Jia, Evan Shelhamer, Jeff Donahue, Sergey Karayev, Jonathan Long, Ross Girshick, Sergio Guadarrama, and Trevor Darrell. Caffe: Convolutional architecture for fast feature embedding. In Proceedings of the 22Nd ACM International Conference on Multimedia, MM '14, pages 675-678, New York, NY, USA, 2014. ACM.

[54] M. Abadi, A. Agarwal, P. Barham, E. Brevdo, Z. Chen, C. Citro, G. S Corrado, A. Davis, J. Dean, M. Devin, S. Ghemawat, I. Goodfellow, A. Harp, G. Irving, M. Isard, Y. Jia, R. Jozefowicz, L. Kaiser, M. Kudlur, J. Levenberg, D. Mane, R. Monga, S. Moore, D. Murray, C. Olah, M. Schuster, J. Shlens, B. Steiner, I. Sutskever, K. Talwar, P. Tucker, V. Vanhoucke, V. Vasudevan, F. Viegas, O. Vinyals, P. Warden, M. Wattenberg, M. Wicke, Y. Yu, and X. Zheng. TensorFlow: Large-Scale Machine Learning on Heterogeneous Distributed Systems. ArXiv eprints, March 2016.
[55] Openblas: An optimized blas library: http://www.openblas.net/. 2017.

[56] Nathan Binkert, Bradford Beckmann, Gabriel Black, Steven K. Reinhardt, Ali Saidi, Arkaprava Basu, Joel Hestness, Derek R. Hower, Tushar Krishna, Somayeh Sardashti, Rathijit Sen, Korey Sewell, Muhammad Shoaib, Nilay Vaish, Mark D. Hill, and David A. Wood. The gem5 simulator. SIGARCH Comput. Archit. News, 39(2):1-7, August 2011.

[57] Alex Krizhevsky. Learning multiple layers of features from tiny images. Technical report, 2009. 